\title{
Energy Expenditure of Two Types of Manual Wheelchair Propulsion
}

\author{
A. Rifai Sarraj ${ }^{* 1,2}$, R. Massarelli ${ }^{1}$, F. Rigal ${ }^{1}$, E. Moussa ${ }^{2}$, C. Jacob², A. Fazah ${ }^{2}$ and M. Kabbara ${ }^{2}$ \\ ${ }^{1}$ Center of Research and Innovation in Sport, Lyon, France \\ ${ }^{2}$ Physiology and Biomechanics of the Motor Performance Laboratory, University of Balamand, Lebanon
}

\begin{abstract}
Objective. To compare the energy expenditure of two types of manual wheelchair propulsion.
Design. Experimental and comparative design - using matched controls.

Background. A new manual wheelchair propulsion technique is proposed to minimize the overload exerted on the upper limb during the classical propulsion. The energy cost and other parameters of this new propulsion technique were evaluated.

Methods. Eight paraplegic male adults (Mean \pm SD) values for age and body mass were $28 \pm 4$ years and $61.75 \pm 8.35 \mathrm{~kg}$ and seven able-bodied subjects (age and body mass were respectively $25 \pm 2$ years and $81.4 \pm 7.4 \mathrm{~kg}$ ) were recruited for this study. Each subject propelled manually two types of wheelchair with two different propulsion techniques. Heart rate and oxygen consumption resulting from each manual propulsion type were calculated and compared.

Results. Mean $\mathrm{VO}_{2}$ peak values were not found to be significantly different between classical and the new proposed wheelchair propulsion for the paraplegic subjects. $\mathrm{VO}_{2}$ values were significantly lower in able-bodied subjects. Maximal Heart Rate were found to be higher when using the classical propulsion technique in both able-bodied and paraplegic groups.

Conclusion. The Classical wheelchair propulsion technique needs to be adapted to the physiological and biomechanical work of the paraplegic's upper extremities. This study showed the advantage of using a different propulsion technique. More studies are needed to recommend the new technique.
\end{abstract}

\section{INTRODUCTION}

Gait is very difficult and mostly impossible following spinal cord injury or any disease affecting the lower limbs. Patients try to find other modalities of physical transport compensating the functional normal gait. Wheelchairs are the most efficient device dedicated to the paraplegic in this case, offering patients the ability to lead a normal life by allowing them to perform most of their daily activities. This ranges from merely being able to relocate from place to place to competing in sporting events.

Wheelchair propulsion has been reported to be responsible for musculoskeletal pain in the upper extremities [1]. Epidemiological studies have shown a high prevalence of shoulder complaints in paraplegic and quadriplegic spinal cord injured (SCI) people [2-8]. It has been argued that the high incidence of shoulder complaints in SCI was the result of the weight-bearing or propulsion function of the upper extremity in those subjects $[9,10]$.

Systematic research has played an important role in the development and design of the wheelchair, vehicle mechanics, the human movement system, and the wheelchairuser interface $[11,12]$.

Variations in propulsion strategy have recently begun to interest researchers [13]. Reverse wheelchair propulsion has been demonstrated to be physiologically more

*Address correspondence to this author at the Center of Research and Innovation in Sport, Lyon, France; E-mail: ahrifpt@hotmail.com demanding than conventional forward wheelchair propulsion [14].

Conventional wheelchair propulsion is commonly divided into two phases, the drive phase and the recovery phase, in each complete propulsive cycle. This type of propulsion is probably limited by a low mechanical efficiency which has been attributed to the position of the arms, synchronicity of the arm movements, a large component of static work, and inherent histochemical characteristics of the upper body musculature [15].

Variations in propulsion techniques becomes a most important issue in patients who use wheelchairs to reduce the incidence of the dysfunction that usually occurs in upper extremities due to the conventional wheelchair propulsion. No variation in propulsion mechanisms has been studied and up to date studies have focused on the conventional wheelchair propulsion technique and its components [16-20].

A proof-of-concept prototype wheelchair with new propulsion technique for the motor disabled will be proposed in the following with the objective to demonstrate the feasibility of a completely new approach to propulsion technique. The aim of this study is to compare the two types of manual wheelchair propulsion technique. The important design considerations and the system design are also discussed.

\section{PROTOTYPE DESIGN}

Our prototype system consists of an adapted wheelchair designed to carry out a different manual wheelchair propulsion technique (Fig. 1). 


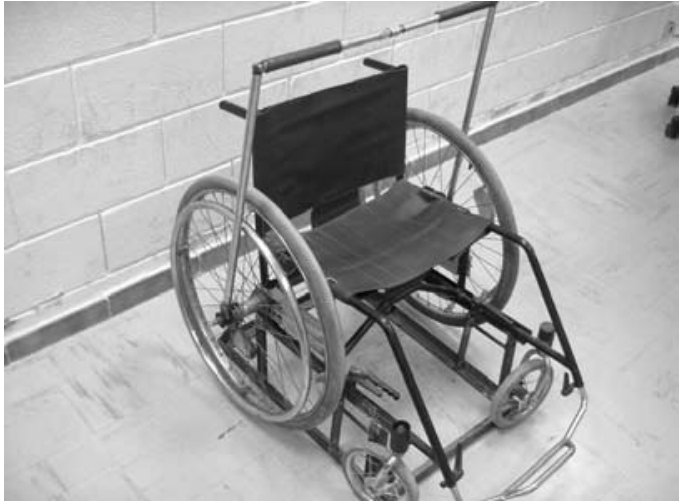

Fig. (1). The new wheelchair prototype.

In mechanical or automotive engineering, a freewheel or overrunning clutch is a device in a transmission that disengages the driveshaft from the driven shaft when the driven shaft rotates faster than the driveshaft. The condition of a driven shaft spinning faster than its driveshaft exists in a bicycle or a vehicle going downhill when the rider holds his or her feet still, no longer pushing the
The same distance traveled by the two types of wheelchairs was found to require the same number of propulsions on both. This is identified by a test realized on basketball court (18 \pm 0.83 propulsions were calculated for both wheelchairs in 8.54 $\pm 0.25 \mathrm{~s}$ on $25 \mathrm{~m})$.

\section{METHODS}

\section{Subjects}

A total of 15 participants were recruited for the study: eight males with paraplegia and seven able-bodied males. For the paraplegic group, the mean $( \pm \mathrm{SD})$ age, height, and weight were respectively $26.9 \pm 6.1$ years, $163.6 \pm 4.2 \mathrm{~cm}$, and $61.4 \pm$ $7.2 \mathrm{~kg}$ (Table 1). For the able-bodied group the values were respectively $24.57 \pm 2$ years, $180.28 \pm 5.79 \mathrm{~cm}$, and $81.42 \pm$ $7.39 \mathrm{Kg}$ (Table 2). Criteria for inclusion were paraplegic with a minimum three years of experience in wheelchair propulsion, and the absence of any medical contra-indications. All subjects signed a written informed consent.

All tests were made in the Laboratory of Physiology and Biomechanics of Motor Performance at the University of

Table 1. Characteristics of the Paraplegic Group

\begin{tabular}{|c|c|c|c|c|c|}
\hline Subject & Age (years) & Height (cm) & Weight (Kg) & Level/Type of Injury & Years in Wheelchair \\
\hline $\mathrm{P} 1$ & 23 & 175 & 71 & $\mathrm{~L} 2$ & 3 \\
\hline $\mathrm{P} 2$ & 34 & 170 & 69 & L1 & 3 \\
\hline P3 & 30 & 172 & 60 & D12 & 8 \\
\hline $\mathrm{P} 4$ & 32 & 169 & 60 & L1 & 14 \\
\hline P5 & 28 & 154 & 55 & D12 & 10 \\
\hline P5 & 30 & 168 & 65 & D12 & 4 \\
\hline P6 & 24 & 165 & 46 & D11 & 8 \\
\hline P7 & 26 & 169 & 68 & L1 & 7 \\
\hline P8 & 23 & 175 & 71 & L2 & 3 \\
\hline Mean \pm SD & $28.38 \pm 3.85$ & $167.75 \pm 6.27$ & $61.75 \pm 8.35$ & & $7.125 \pm 3.80$ \\
\hline
\end{tabular}

pedals. Without a freewheel the rear wheel would drive the pedals round.

The new wheelchair is a classical sport wheelchair equipped with bicycle freewheels attached to the rear wheel hub so the wheel of the wheelchair can go forward with a push of the external part of the freewheel without going backwards when the external part moves in the other direction. The external part is attached to an extended bar manipulated by the hand of the user.

Thus, the two wheels of the classical wheelchair are adapted according to this system and two bars, manipulated by the hands of the user, propel the wheelchair forward.

The new manual wheelchair propulsion technique requires a forward rotating motion by the two bars using the hands of the user imitating the system of the manual press.
Balamand. The protocol was approved by the University Research ethics committee and the procedures complied with the recommendations of the American College of Sports Medicine (ACSM) in 1999. The subjects were told not to practice any exhausting exercise the day before, and not to smoke or drink alcohol $3 \mathrm{~h}$ before the exercise tests. Throughout the exercise they were encouraged to carry out their maximum performances. A general physician was present to support any medical complications during or after the test. The order of the different exercise tests was randomized.

\section{Modeling}

Two wheelchairs (Quickie GT: lightweight non-folding wheelchair) were used in this study. One of the wheelchairs was modified to provide the new propulsion technique discussed above.

The two wheelchairs were raised from the ground by a stand designed to maintain the stability and the free movement 
of the wheels. The coefficient of friction was set to zero for the two propulsion techniques.

Table 2. Characteristics of the Able-Bodied Group

\begin{tabular}{|c|c|c|c|}
\hline Subject & Age (years) & Height (cm) & Weight (Kg) \\
\hline \hline AB1 & 24 & 184 & 85 \\
\hline AB2 & 25 & 177 & 69 \\
\hline AB3 & 25 & 190 & 89 \\
\hline AB4 & 24 & 177 & 85 \\
\hline AB5 & 21 & 184 & 85 \\
\hline AB6 & 29 & 176 & 73 \\
\hline AB7 & 24 & 174 & 84 \\
\hline Mean \pm SD & $24.57 \pm 2$ & $180.28 \pm 5.79$ & $81.42 \pm 7.39$ \\
\hline
\end{tabular}

\section{Testing Protocol}

Subjects that participated in this study were prepared and informed of the protocol methodology prior to the test. This testing protocol consisted of two sessions of manual wheelchair propulsion movements achieved using the two wheelchairs each scheduled on a separate day. The type of the wheelchair in each session was selected by a drawing lot. Each subject was transferred to the selected wheelchair, and the footrests were adjusted to each subject. A metronome was used to fix the rhythm of the testing propulsion.

The duration of each session was seventeen minutes distributed as follows: two minutes of rest, two minutes of warm up, two minutes of preparation to the rhythm, six minutes of rhythmical propulsion, two minutes of maximal number of propulsion movements, one minutes of active recovery and two minutes of passive rest.

During warm up, the subject propelled the selected wheelchair freely using minimal effort. The preparation consisted of two phases of propulsion, each three seconds, and the rhythmical propulsion consisted of a propulsion of two seconds each during the first three minutes and one second each during the second period.

\section{Metabolic Data Analysis}

Oxygen consumption and carbon dioxide production $\left(\mathrm{VCO}_{2}\right)$ were measured breath-by-breath, at rest, during exercise, and throughout recovery using $\mathrm{CPX}^{\circledR}$ Medical Graphics. The system was calibrated before each test session using standard gas mixtures.

The subjects breathe through a mask connected to a pneumotachograph located on the expiratory circuit and connected to a pressure transducer. The quantity of expired oxygen and carbon dioxide were measured, respectively, with a zirconium $\mathrm{O}_{2}$ analyzer and an infrared $\mathrm{CO}_{2}$ analyzer. Before each test the volume was calibrated with a 3-1 pump and the analyzers were standardized with a bottle containing $12 \% \quad \mathrm{O}_{2}$ and $5 \% \mathrm{CO}_{2}$. Peak $\mathrm{VO}_{2}$ data were averaged over 15 seconds intervals. The respiratory exchange ratio (RER) was calculated as $\mathrm{VCO}_{2} / \mathrm{VO}_{2}$.
Heart rate was recorded every minute during exercise and while at rest using a heart rate monitor (POLAR S610i). The wristwatch was positioned on the handle of the wheelchair and the electrode was placed over the manubrium and the left seventh inter-costal space of the subject. Peak levels for all variables were defined as the highest value measured during the exercise test.

\section{Statistical Analysis}

The results are expressed as the mean \pm SD (the standard deviation of the mean) (Tables 3, 4). T-tests were used to assess differences between the energy cost variables of the propulsion of the two wheelchairs. $\mathrm{P}$ values of less than 0.05 were considered to be statistically significant.

Table 3. Mean Values of $\mathrm{VO}_{2}$ Peak and Max Heart Rate for the Paraplegic Group Using Classic Wheelchair (CWC) and New Wheelchair (NWC)

\begin{tabular}{|c|c|c|c|c|}
\hline \multirow{2}{*}{ Paraplegic Subject } & \multicolumn{2}{|c|}{ VO $_{2}$ Peak } & \multicolumn{2}{c|}{ Max Heart Rate } \\
\cline { 2 - 5 } & CWC & NWC & CWC & NWC \\
\hline \hline P1 & 17 & 15.2 & 179 & 123 \\
\hline P2 & 14.2 & 13.6 & 95 & 97 \\
\hline P3 & 19.2 & 18.6 & 133 & 117 \\
\hline P4 & 19.2 & 18.3 & 158 & 109 \\
\hline P5 & 27.6 & 19.6 & 177 & 119 \\
\hline P6 & 14.7 & 10.8 & 153 & 125 \\
\hline P7 & 29.2 & 27.7 & 183 & 185 \\
\hline P8 & 15.6 & 14.4 & 144 & 135 \\
\hline
\end{tabular}

Table 4. Mean values of $\mathrm{VO}_{2}$ Peak and Max Heart Rate for the Able-Bodied Group Using Classic Wheelchair (CWC) and New Wheelchair (NWC)

\begin{tabular}{|c|c|c|c|c|}
\hline \multirow{2}{*}{ Able-bodied Subject } & \multicolumn{2}{|c|}{ VO $_{2}$ Peak } & \multicolumn{2}{c|}{ Max Heart Rate } \\
\cline { 2 - 5 } & CWC & NWC & CWC & NWC \\
\hline \hline AB1 & 25.2 & 22.7 & 123 & 107 \\
\hline AB2 & 21.4 & 20.2 & 173 & 154 \\
\hline AB3 & 23.8 & 19.6 & 167 & 142 \\
\hline AB4 & 23.6 & 17 & 177 & 152 \\
\hline AB5 & 23 & 15.9 & 158 & 132 \\
\hline AB6 & 17.4 & 16 & 165 & 130 \\
\hline AB7 & 20.9 & 18.1 & 145 & 124 \\
\hline
\end{tabular}

\section{Results}

Mean values of $\mathrm{VO}_{2}$ peak were respectively $19.59 \pm 5.76$ and $17.28 \pm 5.14$ for the classic wheelchair and the new wheelchair for the paraplegic group $(p<0.21)$. Mean values of $\mathrm{VO}_{2}$ peak were respectively $22.19 \pm 2.57$ and $18.5 \pm 2.49$ for the classic wheelchair and the new one for the able-bodied subjects with a $\mathrm{p}<0.0092$ (Table 5). 
Table 5. Differences in Mean Values of $\mathrm{VO}_{2}$ Peak between Classic Wheelchair (CWC) and New Wheelchair (NWC) for the Two Groups

\begin{tabular}{|c|c|c|c|}
\hline \multicolumn{4}{|c|}{ VO $_{2}$ Peak (Mean) } \\
\hline & CWC & NWC & p-value \\
\hline \hline Paraplegic & $19.59 \pm 5.76$ & $17.28 \pm 5.14$ & 0.21 \\
\hline $\mathrm{AB}$ & $22.19 \pm 2.57$ & $18.5 \pm 2.49$ & 0.0092 \\
\hline
\end{tabular}

Mean values of Max Heart Rate for the paraplegic group were respectively $152.75 \pm 29.33$ for the classical and $126.25 \pm 26.27$ for the new wheelchair $(\mathrm{p}<0.038)$. For the able-bodied subjects the mean values of Max Heart Rate were respectively $158.29 \pm 18.75$ and $134.43 \pm 16.51$ $(\mathrm{p}<0.013296)$ (Table 6).

There was no difference between the mean values of $\mathrm{VO}_{2}$ peak for the two types of wheelchair in the paraplegic group. However, there was a significant difference between values of $\mathrm{VO}_{2}$ peak for the classic wheelchair and the new one in able-bodied subjects.

Similarly, the values of Max Heart Rate measured during exercise on the two types of wheelchair was significantly different in both groups. Values measured during exercise on the classic wheelchair were higher.

Table 6. Differences in Mean Values of Max Heart Rate between Classic Wheelchair (CWC) and New Wheelchair (NWC) for the Two Groups

\begin{tabular}{|c|c|c|c|}
\hline \multicolumn{4}{|c|}{ Max Heart Rate (Mean) } \\
\hline & CWC & NWC & p-value \\
\hline \hline Paraplegic & $152.75 \pm 29.33$ & $126.25 \pm 26.27$ & 0.038866 \\
\hline AB & $158.29 \pm 18.75$ & $134.43 \pm 16.51$ & 0.013296 \\
\hline
\end{tabular}

\section{Discussion}

Able-bodied subjects showed greater $\mathrm{VO}_{2}$ peak values using the classic wheelchair. These results were not found for the paraplegic subjects. The Max Heart Rate values, recorded during exercises on the classical wheelchair, were higher than values recorded on the new wheelchair for both groups.

The purpose of the experiment was to compare each of the two important parameters related to the energy expenditure for the two types of propulsion techniques. It is proposed that the new prototype was more economical and we hypothesize that the $\mathrm{VO}_{2}$ and the Max Heart Rate must be greater in exercising using the classical technique.

The results further showed that the new propulsion technique is more economical than the classical technique only in able-bodied subjects despite the important differences in Maximal Heart Rate in both groups. A possible explanation may imply the fact that the paraplegic subjects are more accustomed to propelling the classic wheelchair; hence exercising the new prototype has introduced a new motor strategy necessitating more practical learning. It has been reported that practice may refine the movement pattern to approximate more closely the optimal mechanical and physiological adaptations within the constraints of the propulsion task [21]. Exercising a new propulsion technique could influence the energy expenditure during a new motor learning phenomena.

Despite the major improvement in biotechnology, and the recognition of a clear relationship between the classical wheelchair manual propulsion technique and musculoskeletal problems, the factors affecting this relationship remains ambiguous when taking into consideration long-term wheelchair use and sporting activities.

Epidemiological studies have shown a high prevalence of shoulder complaints in paraplegic and quadriplegic spinal cord injured (SCI) people [2]. Several studies calculated joint torques produced around joints of the upper extremity when using classic wheelchair propulsion [22]. Modeling results reported by these authors indicated that the largest joint torques are produced around the shoulder complex. These authors reported peak shoulder flexion torques of $35 \mathrm{Nm}$, in combination with peak adduction torques of $20 \mathrm{Nm}$ [23]. Peak elbow extension torques were $10 \mathrm{Nm}$. They also reported the largest torques around the shoulder joint, but predominantly as a shoulder flexion torque [24]. A large shoulder flexion torque of approximately $30 \mathrm{Nm}$ and elbow extension torque of approximately $10 \mathrm{Nm}$ were also calculated in other study [25]. These data showed the inadaptability and unsuitability of the classical wheelchair propulsion technique for the physiological and biomechanical aspects of the upper extremities of the paraplegic subject.

This study is a trial to develop a new prototype of wheelchair providing a new motor strategy. Parameters related to cardiovascular responses were studied in this article, but other parameters relating to muscles activation strategies, motion data and joint torques must be obtained before recommending this new strategy especially for paraplegic athletes who use the wheelchair in non-physiological conditions.

\section{CONCLUSION}

Patients with paraplegia rely solely on the use of their upper extremities for independence and the completion of daily living activities. Classic wheelchair propulsion is inefficient and not suitable for all physiological biomechanics of the upper extremity joints.

Proposing a new prototype could change the strategy of the classic propulsion technique and may have some advantages particularly in cardiovascular responses, which have been demonstrated during this study.

Future studies questioning dynamic and static parameters related to muscle and movement strategies of the new technique are needed before the new wheelchair propulsion can be recommended with confidence.

\section{REFERENCES}

[1] Boninger M, Dicianno B, Cooper R, Towers J, Koontz A, Souza A. Shoulder magnetic resonance imaging abnormalities, wheelchair propulsion, and gender. Arch Phys Med Rehabil 2003; 84 (16): 15 20 
[2] Sie I, Waters R, Adkins R, Gellman H. Upper extremity pain in the postrehabilitation spinal cord injured patient. Arch Phys Med Rehabil 1992; 73: 44-8.

[3] Dalyan M, Cardenas D, Gerard B. Upper extremity pain after spinal cord injury. Spinal Cord 1999; 37 (3): 191-5.

[4] Van Drongelen S, de Groot S, Veeger HE, et al. Upper extremity musculoskeletal pain during and after rehabilitation in wheelchair-using persons with a spinal cord injury. Spinal Cord 2006; 44: 152-9

[5] Finnerup N, Johannesen I, Sindrup S, Bach F, Jensen T. Pain and dysesthesia in patients with spinal cord injury: a postal survey. Spinal Cord 2001; 39: 256-62

[6] Jensen M, Hoffman A, Cardenas D. Chronic pain in individuals with spinal cord injury: a survey and longitudinal study. Spinal Cord 2005; 43: 704-12.

[7] Widerström-Noga E, Felipe-Cuervo E, Yezierski R. Chronic pain after spinal injury: Interference with sleep and daily activities. Arch Phys Med Rehabil 2001; 82(11): 1571-7.

[8] Widerström-Noga E, Felipe-Cuervo E, Yezierski R. Relationships among clinical characteristics of chronic pain after spinal cord injury. Arch Phys Med Rehabil 2001; 82(9): 1191-7.

[9] Bayley J, Cochran T, Sledge C. The weight-bearing shoulder. The impingement syndrome in paraplegics. J Bone Joint Surg Am 1987; 69(5): 676-8.

[10] Veeger H, van der Woude L. Load on the upper extremity in manual wheelchair propulsion. J Electromyogr Kinesiol 1991; 1: 270-80.

[11] Van der Woude L, de Groot S, Janssen T. Manual wheelchairs: research and innovation in sports and daily life. Sci Sports 2006; 21: 226-35.

[12] Vanlandewijck Y, Theisen D, Daly D. Wheelchair propulsion biomechanics: implications for wheelchair sports. J Sports Med 2001; 31(5): 339-67.

[13] Goosey-Tolfrey VL, Fowler N, Campbell I, Iwnicki S. A kinetic analysis of trained wheelchair racers during two speeds of propulsion. Med Eng Phys 2001; 23: 259-66.
[14] Salvi F, Hoffman M, Sabharwal S, Clifford P. Physiologic comparison of forward and reverse wheelchair propulsion. Arch Phys Med Rehabil 1998; 79: 36-40.

[15] Glaser RM. Exercise and locomotion for the spinal cord injured. Exerc Sport Sci Rev 1985; 13: 263-303.

[16] Rodgers M, Keyser R, Gardner E, Russell P, Gorman P. Influence of trunk flexion on biomechanics of wheelchair propulsion. J Rehabil Res Dev 2000; 37(3): 283-95.

[17] Newsam C, Mulroy S, Gronley J, Bontrager E, Perry J. Temporalspatial characteristics of wheelchair propulsion: effects of level of spinal cord injury, terrain, and propulsion rate. Am J Phys Med Rehabil 1996; 75(4): 292-9.

[18] Kulig K, Rao S, Mulroy S, Newsam C, Gronley J, Bontrager E. Shoulder joint kinetics during the push phase of wheelchair propulsion. Clin Orthop Relat Res 1998; 1(354): 132-43.

[19] Amazeen P, Amazeen E, Beek P. Coupling of breathing and movement during manual wheelchair propulsion. J Exp Psychol Hum Percept Perform 2001; 27(5): 1243-59.

[20] Lan-Yuen G, Fong-Chin S, Hong-Wen W, Kai-Nan A. Mechanical energy and power flow of the upper extremity in manual wheelchair propulsion. Clin Biomech 2003; 18(2): 106-14.

[21] Sparrow WA. The efficiency of skilled performance. J Mot Behav 1983; 15: 237-61.

[22] van der Woude L, Veeger H, Dallmeijer A, Janssen T, Rozendaal L. Biomechanics and physiology in active manual wheelchair propulsion. Med Eng Phys 2001; 23: 713-33.

[23] Veeger HE, van der Woude LH, Rozendal RH. Within cycle characteristics of the wheelchair push in sprinting on a wheelchair ergometer. Med Sci Sport Exerc 1991; 23(2): 264-71.

[24] Wu H, Su F. An instrumented wheel for the kinetic analysis of wheelchair propulsion. J Biomed Eng 1998; 120: 533-5.

[25] Rodgers MM, Tummarakota S. Three-dimensional analysis of wheelchair propulsion. J Appl Biomech 1998; 14: 80-92.

Received: July 01, 2008

Revised: August 01, 2008

Accepted: August 05, 2008

(C) Sarraj et al.; Licensee Bentham Open.

This is an open access article licensed under the terms of the Creative Commons Attribution Non-Commercial License (http://creativecommons.org/licenses/by$\mathrm{nc} / 3.0 /$ ), which permits unrestricted, non-commercial use, distribution and reproduction in any medium, provided the work is properly cited. 